\title{
An Improved Standard Model Comes with Explicit CPV and Productive of BAU
}

\author{
Chilong Lin \\ Department of Exhibition, National Museum of Natural Science, Taiwan \\ Email: lingo@mail.nmns.edu.tw, chilonglin@yahoo.com.tw
}

How to cite this paper: Lin, C.L. (2020) An Improved Standard Model Comes with Explicit CPV and Productive of BAU. Journal of Modern Physics, 11, 1157-1169. https://doi.org/10.4236/jmp.2020.118072

Received: June 30, 2020

Accepted: August 4, 2020

Published: August 7, 2020

Copyright $\odot 2020$ by author(s) and Scientific Research Publishing Inc. This work is licensed under the Creative Commons Attribution International License (CC BY 4.0).

http://creativecommons.org/licenses/by/4.0/

\begin{abstract}
In this manuscript, we present an explicit way to describe the violation of $\mathrm{CP}$ symmetry in the standard model (SM) of electrweak interactions. In such a way, complex Cabbibo-Kobayashi-Maskawa (CKM) matrices are achieved which stand for the violation of CP symmetry. At the beginning, two necessary but not sufficient conditions for yielding a complex CKM matrix are stated as criteria. Then we found an interesting condition between the real and imaginary parts of a Hermitian $3 \times 3$ matrix which may provide extra relations among its parameters and reduce the number of them from eighteen down to five. In previous investigations, this can be done only down to nine. With another assumption among some of those parameters, the mass-matrix pattern is further simplified so as to be diagonalized analytically and in consequence four matrices which reveal $S_{N}$ symmetries among or between quark generations are obtained. In some of such $\mathrm{S}_{\mathrm{N}}$-symmetric cases, the derived CKM matrices are complex which indicate that CP symmetry is violated accordingly. Taking the Jarlskog invariant as an estimate of the CPV strength, the value predicted by this model is orders stronger than the empirical value detected experimentally. However, that happens to fill partly the gap between the cosmologically observed amount of Baryon Asymmetry of the Universe (BAU) and that current Standard Model of particle physics predicts. It also proves the long suspected existence of BAU-productive eras in early universe if some fermions were indistinguishable, i.e., $\mathrm{S}_{\mathrm{N}}$-symmetric, under circumstances with extremely high temperatures.
\end{abstract}

\section{Keywords}

CP Violation (CPV), Cabbibo-Kobayashi-Maskawa (CKM) Matrix, Baryon Asymmetry of the Universe (BAU), $\mathrm{S}_{\mathrm{N}}$ Symmetry

\section{Introduction}

Since the first detection of CP violation (CPV) in 1964 [1], the issue of how CP 
symmetry was violated, attracting our interest very much. However, for over fifty years, an explicit way to describe the violation of CP symmetry is still obscure. If we analyze the theoretical origin of CPV from its fundamental, considering only the quark sector here, the patterns of quark-mass matrices $M$ are obviously the keys to ignite such a violation. Thus, we would like to analyze the CPV problem by starting from the most general pattern of an $M$ matrix and then simplify it to a manageable level step by step.

In the standard model (SM) of electroweak interactions "Direct" CPV is allowed if a complex phase appears in the Cabbibo-Kobayashi-Maskawa (CKM) matrix describing quark mixings, or the Pontecorvo-Maki-Nakagawa-Sakata (PMNS) matrix describing lepton mixings. In SM such a complex phase can only be yielded by ingeniously arranging the Yukawa couplings between fermions and Higgs fields and then diagonalizing the Yukawa-coupling matrix directly. However, after decades such a satisfactory Yukawa-coupling matrix is still obscure.

Theoretically there is another way in SM to extract a complex phase from the vacuum expectation value (VEV) of its only Higgs doublet. However, such a phase can always be absorbed into redefinitions of quark fields in the SM. Thus, an extension of SM with one extra Higgs doublet was proposed in [2] which is usually referred to as the Two-Higgs-doublet model (2HDM). In this way, people expect the phases of two VEVs may unlikely be rotated away simultaneously if there were a nontrivial phase difference between them. However, such an extra Higgs doublet not only failed to solve the CPV problem, but also brought in an extra problem, the flavor-changing neutral current (FCNC) problem at tree level.

In fact, most of the derivations in this manuscript were originally proposed for solving the FCNC problem and CPV problem in the 2HDM [3]. However, we find they apply to the SM as well. As SM alone is enough to describe the violation of CP symmetry, why should one bother to deal with the extra Higgs doublet and problems it brings in? Thus, this manuscript is to be devoted to the theoretical ignition of CPV in the SM alone.

The Yukawa couplings of $Q$ quarks in SM are usually given as

$$
-L_{Y}=\bar{Q}_{L} Y^{d} \Phi d_{R}+\bar{Q}_{L} \epsilon Y^{u} \Phi^{*} u_{R}+\text { h.c. }
$$

where $Y^{q}$ are $3 \times 3$ Yukawa-coupling matrices for quark types $q=u, d$ and $\epsilon$ is the $2 \times 2$ anti-symmetric tensor. $Q_{L}$ is left-handed quark doublets, and $d_{R}$ and $u_{R}$ are right-handed down- and up-type quark singlets in their weak eigenstates, respectively.

When the Higgs doublet $\Phi$ acquires a VEV, $\langle\Phi\rangle=\left(\begin{array}{c}0 \\ \frac{v}{\sqrt{2}}\end{array}\right)$, Equation (1) yields mass terms for quarks with $M^{q}=Y^{q} v / \sqrt{2}$ the mass matrices for $q=u$, $d$. The physical states are obtained by diagonalizing $Y^{q}$ with unitary matrices $U_{L, R}^{q} \quad$ as $\quad M_{\text {diag. }}^{q}=U_{L}^{q} M^{q} U_{R}^{q}=U_{L}^{q} Y^{q} U_{R}^{q}(v / \sqrt{2})$, where $L$ and $R$ are their chirality. 
As a result, the charged-current $W^{ \pm}$interactions couple to the physical $u_{L}$ and $d_{L}$ quarks with couplings given by

$$
-L_{W^{ \pm}}=\frac{g}{\sqrt{2}}\left(\bar{u}_{L}, \bar{c}_{L}, \bar{t}_{L}\right) \gamma^{\mu} W_{\mu}^{+} V_{C K M}\left(\begin{array}{c}
d_{L} \\
s_{L} \\
b_{L}
\end{array}\right)+\text { h.c., }
$$

where

$$
V_{C K M}=U_{L}^{u} \cdot U_{L}^{d \dagger}=\left(\begin{array}{ccc}
V_{u d} & V_{u s} & V_{u b} \\
V_{c d} & V_{c s} & V_{c b} \\
V_{t d} & V_{t s} & V_{t b}
\end{array}\right),
$$

is the CKM matrix describing quark mixings. Hereafter, we will neglect the sub-index $L$ in quark fields $q_{L}$ and the unitary matrices $U_{L}$ if unnecessary.

If one demands a complex CKM matrix, following two conditions are necessary but not sufficient:

Condition 1: At least one of either $U^{u}$ or $U^{d}$ is complex, i.e., $U^{u}$ and $U^{d}$ musn't be both real.

Condition 2: Even if both of them were complex, they musn't be the same, i.e., $U^{u} \neq U^{d}$. Otherwise, $V_{\text {СКM }}$ in Equation (3) will be a $1_{3 \times 3}$ identity matrix which is obviously real.

As $V_{\text {СKM }}=U_{L}^{u} \cdot U_{L}^{d \dagger}$, obviously these two $U$ matrices decide everything of $V_{\text {СKM }}$ including if it were complex. As $U^{u}$ and $U^{d}$ are unitary matrices which diagonalize mass matrices $M^{u}$ and $M^{d}$, respectively. It is obvious they are objects derived from $M^{u}$ and $M^{d}$. Or, we may say the patterns of $M^{u}$ and $M^{d}$ are keys to ignite CPV in the standard model. Thus, we will start the investigation from a most general pattern of $M$ matrix and then put in constraints to see what will happen to the CPV under various circumstances.

In section II, the most general pattern of $M$ matrix is given as a start point of the investigation. If $M$ were Hermitian, an interesting condition Equation (6) comes in if its real and complex parts can be diagonalized respectively by a same unitary matrix and the number of independent parameters in the $M$ matrix is reduced from eighteen down to five. While in previous similar researches this can be done only down to nine with the same assumption.

Since only three quark masses are now given in a quark type, an $M$ matrix with five unknowns is still too complicated to be diagonalized analytically. Thus, an assumption $A=A_{1}=A_{2}=A_{3}$, where $A_{1}, A_{2}$ and $A_{3}$ are diagonal elements of the most general $M$ matrix to be given in section II, is employed to further simplify its pattern. Such an assumption gives us four analytically diagonalizable $M_{k}$ matrices and correspondingly four complex $U_{k}$ matrices, where $k=1$ to 4 indicate to which case they correspond. These multiple $U_{k}$ matrices enable us to satisfy both necessary conditions mentioned above for yielding a complex CKM matrix.

Even we have already a way to describe the violation of CP symmetry within the standard model. However, as to be demonstrated in Section III, the CPV de- 
rived in such $\mathrm{S}_{\mathrm{N}}$-symmetric cases are orders stronger than the value current $\mathrm{SM}$ can provide and orders weaker than the one needed to account for the cosmologically observed Baryon Asymmetry of the Universe (BAU) [and references therein] [4]. The derived Jarlskog invariant $J \sim 0.171$, which is usually used to estimate the CPV strength [5], is about four orders stronger than the experimentally detected value $J=3.00_{-0.09}^{+0.15} \times 10^{-5}$ [6] which corresponds to the BAU amount of order $\eta \sim 10^{-20}$ [7] and at least six orders weaker than the quantity $\eta=\left.\frac{N_{B}}{N_{\gamma}}\right|_{T=3 \mathrm{~K}} \sim 10^{-10}$ [6] [7] [8] estimated from the baryon/photon ratio observed in present universe.

At first glance such a CPV strength looks like a defect of the model. However, if we consider such $S_{N}$ symmetries should exist only under circumstances of extremely high temperatures. It is not strange that CPV derived in this manuscript is different to the one detected in present experiments. Besides, it indicates the BAU we see today could be remnant left over in some early $S_{N}$-symmetric eras of our universe, at least part of them.

Conclusions and discussions are to be given in section IV.

\section{Analytically Diagonalizable $M$ Matrices and Their $U$ Matrices}

As mentioned in Equation (3), $V_{C K M}$ is an inner product of two $U$ matrices, one $U^{u}$ from the up-type quarks and one $U^{d+}$ from the down-type quarks, while they are respectively derived from the mass matrices $M^{u}$ and $M^{d} . \mathrm{Ob}$ viously these two $M$ matrices are key factors to determine if $V_{C K M}$ were complex. Based on this, the most orthodox way is to start from the most general pattern of $M$ matrices and then diagonalize them to achieve corresponding $U$ matrices.

In the SM with three fermion generations and one Higgs doublet, the mass matrix of any specific fermion type must has the general pattern as

$$
M=\left(\begin{array}{ccc}
A_{1}+i D_{1} & B_{1}+i C_{1} & B_{2}+i C_{2} \\
B_{4}+i C_{4} & A_{2}+i D_{2} & B_{3}+i C_{3} \\
B_{5}+i C_{5} & B_{6}+i C_{6} & A_{3}+i D_{3}
\end{array}\right)=\left(\begin{array}{ccc}
A_{1} & B_{1} & B_{2} \\
B_{4} & A_{2} & B_{3} \\
B_{5} & B_{6} & A_{3}
\end{array}\right)+i\left(\begin{array}{ccc}
D_{1} & C_{1} & C_{2} \\
C_{4} & D_{2} & C_{3} \\
C_{5} & C_{6} & D_{3}
\end{array}\right),
$$

which contains eighteen parameters and all $A, B, C$ and $D$ parameters are real.

Theoretically, the most orthodox way to derive the CKM matrix is to diagonalize one such matrix for up-type quarks and one for down-type quarks and then derive their corresponding $U$ matrices. However, such a pattern is obviously too complicated to be diagonalized analytically. Not to mention the $V_{\text {СKM }}$ thus derived contains thirty six parameters in total, eighteen from the up-type quarks and eighteen from the down-type quarks.

In many previous studies, physicists employed various ad hoc constraints to simplify the matrix pattern to a manageable level. For instance, the Fritzsch ansatz (FA) [9] [10] and its subsequent developments like Cheng-Sher ansatz (CSA) [11], Du-Xing ansatz (DXA) [12], combination of the Fritzsch and the 
Du-Xing ansatz (DFXA and FDXA), combination of different assignments in the Du-Xing ansatz ( X̃A), Non-mixing Top quark Ansatz (NTA) [and references therein] [13] and Fukuyama-Nishiura ansatz (FNA) [and references therein] [14] imposed several zeros in the $M$ matrix as ad hoc constraints to reduce the number of parameters in it. The goal of all of them is just to simplify the $M$ pattern to a manageable level. However, constraints so strong are in fact unnatural and unnecessary. As to be demonstrated in what follows, a very weak assumption that $M$ matrices are Hermitian is already enough to yield a very simple $M$ pattern and it includes almost previous ansatz as special cases in it.

It's interesting that action of "different assignments" in [13] and subsequent researches had in fact noticed the necessary condition 2. However, they did not state this condition explicitly like what is done here.

In SM, the Lagrangian as a whole is assumed to be Hermitian. If we assume the Yukawa couplings or equivalently the $M$ matrices were also Hermitian, their patterns will be simplified remarkably. As shown in one of our previous investigations [15], such an assumption can reduce those eighteen parameters in Equation (4) down to only five.

In the first stage, the Hermitian assumption $M=M^{+}$acquires that $D_{j}=0$, $B_{j+3}=B_{j}$ and $C_{j+3}=-C_{j}$, for $j=1,2,3$. Thus, Equation (4) becomes

$$
\begin{aligned}
M & =\left(\begin{array}{ccc}
A_{1} & B_{1}+i C_{1} & B_{2}+i C_{2} \\
B_{1}-i C_{1} & A_{2} & B_{3}+i C_{3} \\
B_{2}-i C_{2} & B_{3}-i C_{3} & A_{3}
\end{array}\right) \\
& =M_{R}+M_{I}=\left(\begin{array}{lll}
A_{1} & B_{1} & B_{2} \\
B_{1} & A_{2} & B_{3} \\
B_{2} & B_{3} & A_{3}
\end{array}\right)+i\left(\begin{array}{ccc}
0 & C_{1} & C_{2} \\
-C_{1} & 0 & C_{3} \\
-C_{2} & -C_{3} & 0
\end{array}\right)
\end{aligned}
$$

where $M_{R}$ and $M_{I}$ are the real and imaginary parts of $M$, respectively. Obviously, $M_{R}$ and $M_{I}$ are also respectively Hermitian. Thus, the number of parameters in $M$ is reduced from eighteen down to nine at this stage.

If there were a unitary matrix $U$ which diagonalize $M_{R}$ and $M_{I}$ simultaneously and respectively, it must also diagonalize the whole $M$ matrix and leads to the following equation which was originally proposed in [16] and revised in [15],

$$
M_{R} M_{I}^{\dagger}-M_{I} M_{R}^{\dagger}=0
$$

Such an equation will provide us several extra relations among parameters and reduce the number of independent parameters further.

Substituting Equation (5) into Equation (6), we receive four equations

$$
\begin{gathered}
B_{1} C_{1}=-B_{2} C_{2}=B_{3} C_{3}, \\
\left(A_{1}-A_{2}\right)=\frac{B_{3} C_{2}+B_{2} C_{3}}{C_{1}}, \\
\left(A_{3}-A_{1}\right)=\frac{B_{1} C_{3}-B_{3} C_{1}}{C_{2}}, \\
\left(A_{2}-A_{3}\right)=-\frac{B_{2} C_{1}+B_{1} C_{2}}{C_{3}} .
\end{gathered}
$$


which will reduce the parameter number from nine further down to five.

However, five parameters are still too many to diagonalize the $M$ matrix analytically. In order to simplify the pattern of $M$ further, we will employ an extra assumption

$$
A=A_{1}=A_{2}=A_{3},
$$

among the diagonal elements of $M$ and further summarize Equations (7)-(10) in

$$
B_{1}^{2}=B_{2}^{2}=B_{3}^{2}, C_{1}^{2}=C_{2}^{2}=C_{3}^{2} .
$$

By examining all possible solutions of Equation (12) we found only four cases satisfy them. In what follows they will be studied respectively as:

Case 1: $B_{1}=B_{2}=B_{3}=B$ and $C_{1}=-C_{2}=C_{3}=C$

In this case,

$$
M_{1}=M_{R}+M_{I}=\left(\begin{array}{ccc}
A & B & B \\
B & A & B \\
B & B & A
\end{array}\right)+i\left(\begin{array}{ccc}
0 & C & -C \\
-C & 0 & C \\
C & -C & 0
\end{array}\right) \text {, }
$$

which has the same pattern as what was achieved in [3] [17] with a $S_{3}$ permutation symmetry imposed among the Yukawa interactions of three quark generations. That symmetry was originally employed to solve the FCNC problem in a 2HDM. However, we find it applies to SM also.

Analytically, the $M$ matrix is diagonalized and the mass eigenvalues are given as

$$
M_{\text {diag. }}=\left(\begin{array}{ccc}
A-B-\sqrt{3} C & 0 & 0 \\
0 & A-B+\sqrt{3} C & 0 \\
0 & 0 & A+2 B
\end{array}\right),
$$

and the $U$ matrix which diagonalize Equation (13) is then given as

$$
U_{1}=\left(\begin{array}{ccc}
\frac{-1-i \sqrt{3}}{2 \sqrt{3}} & \frac{-1+i \sqrt{3}}{2 \sqrt{3}} & \frac{1}{\sqrt{3}} \\
\frac{-1+i \sqrt{3}}{2 \sqrt{3}} & \frac{-1-i \sqrt{3}}{2 \sqrt{3}} & \frac{1}{\sqrt{3}} \\
\frac{1}{\sqrt{3}} & \frac{1}{\sqrt{3}} & \frac{1}{\sqrt{3}}
\end{array}\right),
$$

where the sub-index $k(k=1$ to 4$)$ of $U_{k}$ indicates to which case it corresponds.

Case 2: $B_{1}=B_{2}=-B_{3}=B$ and $C_{1}=-C_{2}=-C_{3}=C$

In this case,

$$
M_{2}=M_{R}+M_{I}=\left(\begin{array}{ccc}
A & B & B \\
B & A & -B \\
B & -B & A
\end{array}\right)+i\left(\begin{array}{ccc}
0 & C & -C \\
-C & 0 & -C \\
C & C & 0
\end{array}\right) \text {, }
$$

which reveals a residual $S_{2}$ symmetry between the second and third generations.

The mass eigenvalues are given as 


$$
M_{\text {diag. }}=\left(\begin{array}{ccc}
A+B-\sqrt{3} C & 0 & 0 \\
0 & A+B+\sqrt{3} C & 0 \\
0 & 0 & A-2 B
\end{array}\right),
$$

and corresponding $U$ matrix is given as

$$
U_{2}=\left(\begin{array}{ccc}
\frac{1-i \sqrt{3}}{2 \sqrt{3}} & \frac{-1-i \sqrt{3}}{2 \sqrt{3}} & \frac{1}{\sqrt{3}} \\
\frac{1+i \sqrt{3}}{2 \sqrt{3}} & \frac{-1+i \sqrt{3}}{2 \sqrt{3}} & \frac{1}{\sqrt{3}} \\
\frac{-1}{\sqrt{3}} & \frac{1}{\sqrt{3}} & \frac{1}{\sqrt{3}}
\end{array}\right) .
$$

Case 3: $B_{1}=-B_{2}=B_{3}=B$ and $C_{1}=C_{2}=C_{3}=C$

In this case,

$$
M_{3}=M_{R}+M_{I}=\left(\begin{array}{ccc}
A & B & -B \\
B & A & B \\
-B & B & A
\end{array}\right)+i\left(\begin{array}{ccc}
0 & C & C \\
-C & 0 & C \\
-C & -C & 0
\end{array}\right),
$$

which reveals a residual $S_{2}$ symmetry between the first and third generations.

The mass eigenvalues are now given as

$$
M_{\text {diag. }}=\left(\begin{array}{ccc}
A+B-\sqrt{3} C & 0 & 0 \\
0 & A+B+\sqrt{3} C & 0 \\
0 & 0 & A-2 B
\end{array}\right),
$$

and corresponding $U$ matrix is given as

$$
U_{3}=\left(\begin{array}{ccc}
\frac{-1+i \sqrt{3}}{2 \sqrt{3}} & \frac{1+i \sqrt{3}}{2 \sqrt{3}} & \frac{1}{\sqrt{3}} \\
\frac{-1-i \sqrt{3}}{2 \sqrt{3}} & \frac{1-i \sqrt{3}}{2 \sqrt{3}} & \frac{1}{\sqrt{3}} \\
\frac{1}{\sqrt{3}} & \frac{-1}{\sqrt{3}} & \frac{1}{\sqrt{3}}
\end{array}\right) .
$$

Case 4: $B_{1}=-B_{2}=-B_{3}=-B$ and $C_{1}=C_{2}=-C_{3}=-C$.

In this case,

$$
M_{4}=M_{R}+M_{I}=\left(\begin{array}{ccc}
A & -B & B \\
-B & A & B \\
B & B & A
\end{array}\right)+i\left(\begin{array}{ccc}
0 & -C & -C \\
C & 0 & C \\
C & -C & 0
\end{array}\right),
$$

which reveals a residual $S_{2}$ symmetry between the first and second generations.

The mass eigenvalues are given as

$$
M_{\text {diag. }}=\left(\begin{array}{ccc}
A+B-\sqrt{3} C & 0 & 0 \\
0 & A+B+\sqrt{3} C & 0 \\
0 & 0 & A-2 B
\end{array}\right),
$$

and corresponding $U$ matrix is then given as 


$$
U_{4}=\left(\begin{array}{ccc}
\frac{1-i \sqrt{3}}{2 \sqrt{3}} & \frac{1+i \sqrt{3}}{2 \sqrt{3}} & \frac{1}{\sqrt{3}} \\
\frac{1+i \sqrt{3}}{2 \sqrt{3}} & \frac{1-i \sqrt{3}}{2 \sqrt{3}} & \frac{1}{\sqrt{3}} \\
\frac{1}{\sqrt{3}} & \frac{-1}{\sqrt{3}} & \frac{1}{\sqrt{3}}
\end{array}\right) .
$$

In all four cases the $U_{k}$ matrices are complex which satisfy the first condition stated in section I. If we assign different of them to up- and down-type quarks respectively, the second condition will also be satisfied. Even so, we are still not sure if $V_{C K M}$ were complex since the inner product of two complex matrices can still be real, which is to be demonstrated in next section.

In fact, derivations presented above were originally proposed to solve the FCNC problem in the $2 \mathrm{HDM}$ by finding matrix pairs which can be diagonalized by a same $U$ matrix simultaneously [18]. However, we found in some special cases the derived CKM matrices are complex which mean that CP symmetry is violated. Besides, the derivations apply not only to $2 \mathrm{HDMs}$, but to the SM also. As $\mathrm{SM}$ is already enough to give CPV explicitly, why should we bother ourselves to deal with the FCNC problem in the 2HDM? Surely we can still apply them to the extensions of SM with one or even two extra Higgs doublets while the FCNC problem vanishes naturally at tree level. But that is a different subject to be discussed elsewhere.

Besides, it is noteworthy those $\mathrm{S}_{\mathrm{N}}$ symmetries are not imposed ad hoc constraints. They are by-products brought in by the assumption among $A$ parameters in Equation (11). Among them, the $S_{3}$-symmetric pattern in case 1 is exactly the same as the one derived in our very early manuscripts [3] [17]. It solved the FCNC problem at tree level successfully but not the CPV problem. The problem it met was the breach of condition $2, U^{u} \neq U^{d}$, since we had only one $\mathrm{S}_{3}$-symmetric $U_{1}$ matrix for both quark types at that time. While in this manuscript, extra $\mathrm{S}_{2}$-symmetric $U_{2}, U_{3}$ and $U_{4}$ matrices provide us a key to ignite violation of CP symmetry from the theoretical end.

\section{CKM Matrix and the BAU Problem}

As mentioned in section I, if one expects to yield a CP-violating phase in the CKM matrix, two necessary conditions are to be satisfied. In last section, four complex $U_{k}$ matrices were achieved with a Hermitian assumption of $M$ and an assumption among $A$ parameters. If we assign different $U_{k}$ matrices to up- and down-type quarks respectively, both conditions are satisfied. In what follows, various assignments of $U_{k}^{u}$ and $U_{k}^{d}$ are examined and $V_{C K M}$ they yield are presented in Table 1 . Several of them are complex which indicate a theoretical origin of CPV is explicitly yielded.

The full expressions of matrices $1_{3 \times 3}, E, F, G$ and $H$ in Table 1 are given as what follows 
Table 1. Various assembles of CKM matrix.

\begin{tabular}{ccccc}
\hline$V_{\text {СКM }}$ & $U_{1}^{d \dagger}$ & $U_{2}^{d \dagger}$ & $U_{3}^{d \dagger}$ & $U_{4}^{d \dagger}$ \\
\hline$U_{1}^{u}$ & $1_{3 \times 3}$ & $E$ & $E^{*}$ & $G$ \\
$U_{2}^{u}$ & $E^{*}$ & $1_{3 \times 3}$ & $H$ & $F$ \\
$U_{3}^{u}$ & $E$ & $H$ & $1_{3 \times 3}$ & $F^{*}$ \\
$U_{4}^{u}$ & $G$ & $F$ & $F^{*}$ & $1_{3 \times 3}$ \\
\hline
\end{tabular}

$$
\begin{aligned}
& 1_{3 \times 3}=\left(\begin{array}{ccc}
1 & 0 & 0 \\
0 & 1 & 0 \\
0 & 0 & 1
\end{array}\right), G=\left(\begin{array}{ccc}
\frac{2}{3} & \frac{-1}{3} & \frac{2}{3} \\
\frac{-1}{3} & \frac{2}{3} & \frac{2}{3} \\
\frac{2}{3} & \frac{2}{3} & \frac{-1}{3}
\end{array}\right), H=\left(\begin{array}{ccc}
\frac{-1}{3} & \frac{2}{3} & \frac{2}{3} \\
\frac{2}{3} & \frac{-1}{3} & \frac{2}{3} \\
\frac{2}{3} & \frac{2}{3} & \frac{-1}{3}
\end{array}\right) \text {, } \\
& E=\left(\begin{array}{ccc}
\frac{1-i \sqrt{3}}{3} & \frac{1}{3} & \frac{1+i \sqrt{3}}{3} \\
\frac{1}{3} & \frac{1+i \sqrt{3}}{3} & \frac{1-i \sqrt{3}}{3} \\
\frac{1+i \sqrt{3}}{3} & \frac{1-i \sqrt{3}}{3} & \frac{1}{3}
\end{array}\right)=\left(\begin{array}{ccc}
\frac{2}{3} \mathrm{e}^{-i \frac{\pi}{6}} & \frac{1}{3} & \frac{2}{3} \mathrm{e}^{i \frac{\pi}{6}} \\
\frac{1}{3} & \frac{2}{3} \mathrm{e}^{i \frac{\pi}{6}} & \frac{2}{3} \mathrm{e}^{-i \frac{\pi}{6}} \\
\frac{2}{3} \mathrm{e}^{i \frac{\pi}{6}} & \frac{2}{3} \mathrm{e}^{-i \frac{\pi}{6}} & \frac{1}{3}
\end{array}\right), \\
& F=\left(\begin{array}{ccc}
\frac{1}{3} & \frac{1-i \sqrt{3}}{3} & \frac{1+i \sqrt{3}}{3} \\
\frac{1+i \sqrt{3}}{3} & \frac{1}{3} & \frac{1-i \sqrt{3}}{3} \\
\frac{1-i \sqrt{3}}{3} & \frac{1+i \sqrt{3}}{3} & \frac{1}{3}
\end{array}\right)=\left(\begin{array}{ccc}
\frac{1}{3} & \frac{2}{3} \mathrm{e}^{-i \frac{\pi}{6}} & \frac{2}{3} \mathrm{e}^{i \frac{\pi}{6}} \\
\frac{2}{3} \mathrm{e}^{i \frac{\pi}{6}} & \frac{1}{3} & \frac{2}{3} \mathrm{e}^{-i \frac{\pi}{6}} \\
\frac{2}{3} \mathrm{e}^{-i \frac{\pi}{6}} & \frac{2}{3} \mathrm{e}^{i \frac{\pi}{6}} & \frac{1}{3}
\end{array}\right),
\end{aligned}
$$

The matrices $1_{3 \times 3}, G$ and $H$ are purely real and obviously CP-conserving. While $E, F$ and their complex conjugates are complex, which means they are CP-violating. It is interesting that as shown in the $G$ and $H$ cases, even if both conditions were satisfied, $V_{C K M}$ is still completely real.

We now have already a way to describe the violation of CP symmetry from the theoretical end. But the CKM elements derived in Equation (25) are very different to experimentally detected corresponding values. Some of them are hundreds times the detected values, say both predicted $\left|V_{u b}\right|=2 / 3$ in $E$ and $F$ are about 184 times the presently detected value $3.82 \pm 0.24 \times 10^{-3} \quad$ [6]. Besides, the CKM matrices derived here contain only numbers rather than any variable factors. That leaves us no space to improve the fitting between theoretical predictions and experimental detections further. This could be ascribed to the over-simplified matrix patterns caused by the $A=A_{1}=A_{2}=A_{3}$ assumption in Equation (11) or equivalently the revealed $S_{N}$ symmetries. However, it may also hint that if we can diagonalize Equation (5) directly rather than imposing the assumption in Equation (11), we will achieve $M$ patterns more close to the ones 
observed now.

As we have already yield several complex $V_{\text {СКM }}$ demonstrated in Table 1, it is rational for us to go one step further to estimate the CPV strength predicted by such a model. In usual, the strength of direct CPV is estimated by the dimensionless Jarlskog determinant [4] [5] [19] [20] which were given as

$$
\begin{aligned}
\Delta_{C P} & =v^{-12} \operatorname{ImDet}\left[m_{u} m_{u}^{\dagger}, m_{d} m_{d}^{\dagger}\right] \\
& =J v^{-12} \prod_{i<j}\left(\tilde{m}_{u, i}^{2}-\tilde{m}_{u, j}^{2}\right) \prod_{i<j}\left(\tilde{m}_{d, i}^{2}-\tilde{m}_{d, j}^{2}\right) \cong 10^{-19},
\end{aligned}
$$

where $v$ is the Higgs vacuum expectation value and $\tilde{m}$ are particle masses.

The Jarlskog invariant " $J$ " in Equation (26) is a phase-convention-independent measure of CPV defined by

$$
\operatorname{Im}\left[V_{i j} V_{k l} V_{i l}^{*} V_{k j}^{*}\right]=J \sum_{m, n} \epsilon_{i k m} \epsilon_{j l n},
$$

and a global fit of its value was given by the Particle Data Group as $J=3.00_{-0.09}^{+0.15} \times 10^{-5}$ [6]. Such a value corresponds to a BAU of the order $\eta \sim 10^{-20}$ if one substitutes the detected fermion masses into Equation (26). It is obviously too small to account for the cosmologically observed

$$
\eta=\left.\frac{N_{B}}{N_{\gamma}}\right|_{T=3 \mathrm{~K}} \sim 10^{-10}
$$

where $N_{B}$ is the number of baryons and $N_{\gamma}$ is the number of photons.

Substituting the values of $V_{c d}, V_{t s}, V_{c b}^{*}$ and $V_{u s}^{*}$ given in Equation (25) into (27), the $J$ values for $F$ and $F^{*}$ are the same

$$
J_{F, F^{*}}=\frac{16}{81} \sin \left(\frac{2 \pi}{3}\right) \sim 0.171,
$$

while those for $E$ and $E^{*}$ are zero since the phases in them are canceled. The $J$ value given in Equation (28) is almost four orders higher than the one given by current SM. It hints that under circumstances with $S_{2}^{(c \leftrightarrow t)}+S_{2}^{(d \leftrightarrow s)}$, $S_{2}^{(u \leftrightarrow t)}+S_{2}^{(d \leftrightarrow s)}, \quad S_{2}^{(u \leftrightarrow c)}+S_{2}^{(d \leftrightarrow b)}$ and $S_{2}^{(u \leftrightarrow c)}+S_{2}^{(s \leftrightarrow b)}$ symmetries, the hyper-indices in the brackets indicate between which two quarks the $S_{2}$ symmetry appears, the CPV strengths thus derived are orders stronger than what we see nowadays and thus more productive of BAU.

But, as constrained by Equation (27), the value of $J$ is always smaller than 1 . So a large $J$ is always not enough to account for all the discrepancy between the cosmologically observed BAU and the one current SM predicts. One another potential source of a large $\Delta_{C P}$ is the mass-depending terms $\left(\tilde{m}_{u, i}^{2}-\tilde{m}_{u, j}^{2}\right)$ and $\left(\tilde{m}_{d, i}^{2}-\tilde{m}_{d, j}^{2}\right)$ in Equation (26). Unfortunately, the mass eigenvalues derived here give no helps on this subject since they are fixed to the detected quark masses. Obviously, finding mass eigenvalues running with some variables in such models will be very helpful in solving the BAU problem.

Besides, symmetries usually exist under circumstances with higher temperatures. As we do not see such $S_{N}$ symmetries among fermion generations in our present universe, it is natural for us to consider they could have appeared in 
some early eras if the Big-Bang cosmology were correct. Thus, the discrepancy between the $\mathrm{S}_{\mathrm{N}}$-symmetric CPV and that detected in present experiments is very natural.

In another way, we may imagine in some very early stages of the universe with extremely high temperature $T$ there were $S_{3}$ symmetries in both quark types. As $T$ decreased with the expansion, some fermions degenerated from others and the symmetries were broken down to a residual $S_{2}$-one. Probably the up-type quarks first and then the down-type quarks followed, but this is not necessary. During a succession of breaking down of $\mathrm{S}_{\mathrm{N}}$ symmetries, for instance

$$
\mathrm{S}_{3}^{u}+S_{3}^{d} \rightarrow S_{2}^{u}+S_{3}^{d} \rightarrow S_{2}^{u}+S_{2}^{d} \rightarrow \mathrm{No}^{u}+S_{2}^{d} \rightarrow \mathrm{No}^{u}+\mathrm{No}^{d}
$$

where the super-indices $u$ and $d$ indicate up- and down-type quarks respectively. At least the $S_{2}^{u}+S_{2}^{d}$ era was proved to be CP-violating and very productive of BAU in this manuscript.

As we do not see any $\mathrm{S}_{\mathrm{N}}$ symmetries in our present universe, obviously we are now in the latest stage, $N o^{u}+N o^{d}$, of Equation (29). That means a completely analytical diagonalization of $M$ matrices is desired. We expect such a diagonalization will give us a $V_{C K M}$ coincides the experimentally detected values better. Unfortunately, this is still unaccomplished for now and will be the next goal of our future investigations.

\section{Conclusions and Discussions}

Since the discovery of CPV in decays of neutral kaons, its theoretical origin is always a puzzle physicists' urgent to solve. Through the analysis on compositions of $V_{C K M}$ two necessary but not sufficient conditions for yielding a complex $V_{C K M}$ are stated and an explicit way to describe the violation of CP symmetry in SM is presented.

The way performed in this manuscript is not a new one to study the CP problem. It's in fact an old and orthodox way in SM, by diagonalizing the $M$ matrices to obtain corresponding $U$ matrices and then yields a complex $V_{\text {СКM }}$. In previous attempts we failed in this goal since most researchers ignored the second condition while in this manuscript that condition is satisfied since four such $U_{k}$ matrices are achieved.

The key factor enables us to achieve a manageable $M$ pattern and consequently a CP-violating $V_{C K M}$ is Equation (6). Such a condition between the real and imaginary parts of a Hermitian matrix is always true as proved in [15] if they were diagonalized by a same $U$ matrix. It correlates the elements in an $M$ matrix and thus reduces the number of parameters from eighteen down to five. Furthermore, with an extra assumption among its parameters as given in Equation

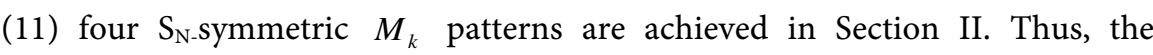
second condition is satisfied and several complex CKM patterns come in explicitly.

Though the magnitudes of Jarlskog invariants thus derived are about four orders that predicted by current SM. However, as we do not see any such $S_{N}$ 
symmetries in our present universe and they should exist only under circumstances with extremely high temperature. It's natural to consider their appearances in some very early stages of the universe. That hints our universe could have been very productive of BAU in such $S_{N_{-}}$symmetric stages. While, as the $S_{N}$ symmetries were broken down completely as the universe expanded, such a BAU-productive mechanism was narrowed down to the present status, $J=\left(3.00_{-0.09}^{+0.15}\right) \times 10^{-5}$. Obviously, such an improved SM not only describes how CP symmetry was violated from the theoretical end, but also explains why the amount of BAU observed today is so huge while the currently detected CPV is so tiny.

This work is not a new model. Instead, it is just an improvement of SM in the sector of fermions' Yukawa interactions. Though it does not solve the CPV and BAU problems completely, it still improves our understanding of the origin of CPV and exhibits a way to account for partly the cosmologically observed BAU which cannot be accounted for by current SM. Such an interesting aspect could be a good stepping stone for coming researchers on these topics.

\section{Conflicts of Interest}

The author declares no conflicts of interest regarding the publication of this paper.

\section{References}

[1] Christenson, J.H., Cronin, J.W., Fitch, V.L. and Turlay, R. (1964) Physical Review Letters, 13, 138-140. https://doi.org/10.1103/PhysRevLett.13.138

[2] Lee, T.D. (1973) Physical Review D, 8, 1226-1239. https://doi.org/10.1103/PhysRevD.8.1226

[3] Lin, C.L., Lee, C.E. and Yang, Y.W. (1988) Chinese Journal of Physics, 26, 180-193.

[4] Tranberg, A., Hernandez, A., Konstandin, T. and Schmidt, M. (2010) Physics Letters B, 690, 207-212. https://doi.org/10.1016/j.physletb.2010.05.030

[5] Jarlskog, C. (1985) Physical Review Letters, 55, 1039-1042. https://doi.org/10.1103/PhysRevLett.55.1039

[6] Zyla, P.A., et al. (2020) Progress of Theoretical and Experimental Physics, 2020, $083 \mathrm{C} 01$.

[7] Canetti, L., Drewes, M. and Shaposhnikov, M. (2012) New Journal of Physics, 14, Article ID: 095012. https://doi.org/10.1088/1367-2630/14/9/095012

[8] Ade, P.A.R., et al. (2016) Astronomy \& Astrophysics, 594, A13.

[9] Fritzsch, H. (1978) Physics Letters B, 73, 317-322. https://doi.org/10.1016/0370-2693(78)90524-5

[10] Fritzsch, H. (1979) Nuclear Physics B, 155, 189-207. https://doi.org/10.1016/0550-3213(79)90362-6

[11] Cheng, T.P. and Sher, M. (1987) Physical Review D, 35, 3484-3491. https://doi.org/10.1103/PhysRevD.35.3484

[12] Du, D. and Xing, Z.Z. (1993) Physical Review D, 48, 2349-2352. https://doi.org/10.1103/PhysRevD.48.2349 
[13] Carcamo, A., Martinez, R. and Rodriguez, J. (2007) The European Physical Journal C, 50, 935-948. https://doi.org/10.1140/epjc/s10052-007-0264-0

[14] Matsuda, K., Nishiura, H. and Fukuyama, T. (2000) Physical Review D, 61, Article ID: 053001. https://doi.org/10.1103/PhysRevD.61.053001

[15] Lin, C.L. (2019) Journal of Modern Physics, 10, 35-42. https://doi.org/10.4236/jmp.2019.101004

[16] Branco, G.C., Buras, A.J. and Gerard, J.M. (1985) Nuclear Physics B, 259, 306-330. https://doi.org/10.1016/0550-3213(85)90638-8

[17] Lee, C.E., Lin, C.L. and Yang, Y.W. (1990) Physical Review D, 42, 2355-2358. https://doi.org/10.1103/PhysRevD.42.2355

[18] Lin, C.L. (2013) Type-IV Two-Higgs-Doublet Models and Their CP-Violation. (Unpublished) arXiv:1308.6039 Lin, C.L. (2014) A Different Origin of CP-Violation in Two-Higgs-Doublet Models. (Unpublished) arXiv:1403.3482

[19] Farrar, G.R. and Shaposhnikov, M.E. (1993) Physical Review Letters, 70, 2833-2836. https://doi.org/10.1103/PhysRevLett.70.2833

[20] Farrar, G.R. and Shaposhnikov, M.E. (1994) Physical Review D, 50, 774-818. https://doi.org/10.1103/PhysRevD.50.774 